Research Article

\title{
Neurospora crassa mat A-2 and mat A-3 proteins weakly interact in the yeast two-hybrid system and affect yeast growth
}

\author{
Carla C. da Silva, Rosana C. Cruz, Mônica Bucciarelli-Rodriguez and Adlane Vilas-Boas \\ Departamento de Biologia Geral, Instituto de Ciências Biológicas, Universidade Federal de Minas Gerais, \\ Belo Horizonte, MG, Brazil.
}

\begin{abstract}
Mating-type genes control the entry into the sexual cycle, mating identity and sexual development in fungi. The mat $A-2$ and mat $A-3$ genes, present in the mat $A$ idiomorph of the filamentous fungus Neurospora crassa, are required for post-fertilization functions but are not essential for mating identity. Their putative roles as transcription factors are based on the similarity of mat $A-2$ with the Podospora anserina SMR1 gene and an HMG motif present in the mat $A-3$ gene. In this work the yeast two-hybrid system was used to identify transcriptional activity and protein-protein interaction of $N$. crassa mat $A-2$ and mat $A-3$ genes. We observed that the mat A-3 protein alone is capable of weakly activating transcription of yeast reporter genes; it also binds with low specificity to the GAL1 promoter sequence, possibly due to its HMG domain. Our results also indicate that mat A-3 is capable to form homodimers, and interact with mat A-2. Interference on yeast growth was observed on some transformants suggesting a toxic action of the mat A-2 protein. Our data on pattern of interactions of mat proteins contributes towards understanding the control of vegetative and sexual cycles in filamentous fungi.
\end{abstract}

Key words: Neurospora crassa, mating-type, yeast two-hybrid system.

Received: November 25, 2008; Accepted: February 3, 2009.

\section{Introduction}

Entry into the sexual cycle in fungi is controlled by the mating-type (mat) genes which are responsible for sexual development and identity in filamentous fungi and yeast (reviewed in Kronstad and Staben, 1997; Souza et al., 2003). Although it is known that many mat gene products are transcription factors, very little is known about how MAT proteins interact and what are the target genes in filamentous ascomycete fungi.

$N$. crassa was the first filamentous fungus that had its mat sequences cloned and physically characterized (Glass et al., 1988). The mat $A$ and mat $a$ are non-homologous sequences present at the mat locus, thus called idiomorphs (Metzenberg and Glass, 1990). The mat a idiomorph contains the mat a-1 gene which is responsible for sexual identity, vegetative incompatibility and perithecium development (Staben and Yanofsky, 1990). The mat a-1 gene has a DNA-binding HMG motif, found in certain transcriptional regulatory proteins, and was shown to regulate the $m f a-1$ pheromone precursor gene among other genes (Kim et al., 2002). A second ORF, mat $a-2$, is also present in the mat a idiomorph, but is apparently not transcribed (Pöggeler and

Send correspondence to Adlane Vilas-Boas Ferreira. Laboratório de Genética de Microrganismos, Departamento de Biologia Geral, Universidade Federal de Minas Gerais, Av. Antônio Carlos 6627, 1270-901 Belo Horizonte, MG, Brazil. E-mail: adlane@ ufmg.br.
Kuck, 2000). The mat $A$ idiomorph contains three genes: mat A-1, mat A-2 and mat A-3 (Glass et al., 1990; Ferreira et al., 1996). The mat $A-1$ gene product contains an $\alpha 1$ domain, similar to Saccharomyces cerevisiae MAT $\alpha 1$ protein. The mat A-1 protein is sufficient and required for vegetative incompatibility, sexual identity, sexual development and production of A-specific pheromones and a-pheromone receptors (Glass et al., 1990; Bobrowicz et al., 2002). Ferreira et al. $(1996,1998)$ have characterized the additional two $N$. crassa mat genes: mat $A-2$ codes for a novel putative DNA-binding domain protein, and mat $A-3$, as the mat $a-1$ gene, codes for an HMG domain protein. The mat $A-2$ and mat $A-3$ genes are similar to the SMR1 and $S M R 2$ mat genes of $P$. anserina, respectively (Ferreira et al., 1996), which are necessary for post-fertilization events in this fungus (Debuchy et al., 1993). Despite the similarity, $N$. crassa mat genes are not capable of complementing P. anserina SMR1 and SMR2 mutants for post-fertilization events (Arnaise et al., 1993). Also, phenotypic analysis of mat $A-2$ and mat $A-3$ mutants indicated that these genes function differently from their homologues (Ferreira et al., 1998), since a mutant phenotype is only observed when both genes are mutated. A question that arises from this intriguing observation is whether mat A-2 and mat A-3 act independently or an interaction between them is needed for their function. 
Protein-protein interaction in the yeast two-hybrid (Y2H) system has been observed between $N$. crassa mat A-1 and mat a-1 proteins (Badgett and Staben, 1999) and between $P$. anserina FMR1 and SMR2 proteins (Debuchy and Coppin, unpublished data cited in Coppin et al., 1997). The four mat genes of the homothallic pyrenomycete Sordaria macrospora (Smta-1, SmtA-1, SmtA-2 and SmtA-3) are all highly similar to $N$. crassa mat genes (Pöggeler et al., 1997). The yeast two-hybrid system was also used to demonstrate that Smta-1 and SmtA-1 proteins interact and that these proteins contain domains capable of activating transcription in yeast (Jacobsen et al., 2002). However, molecular interaction data on the $N$. crassa mat $A-2$ and mat $A-3$ gene products or their homologues are not available. In this work we used the Y2H system to verify possible protein-protein interactions and transcriptional activation activity of the $N$. crassa mat $A-2$ and mat $A-3$ gene products.

\section{Materials and Methods}

\section{Strains and media}

Saccharomyces cerevisiae HF7c (Mat a, ura3-52, his3-200, lys2-801, ade2-101, trp1-901, leu2-3/112, gal4542, gal80-538, LYS2::GAL1-HIS3, URA3:(GAL4 17mer $_{3}$ )-CYC-lacZ) and Y190 (MATa trp1-901 his3 leu23,112 ura3-52 ade2 gal4 gal80 URA3::GAL1-lacZ LYS2::GAL1-HIS3) strains (Clontech Laboratories, Inc.) were used for the two-hybrid experiments. Isogenic IH 1783 (MAT a trp1 leu2 ura3 his4 can1), IH1784 (MAT $\alpha$ trpl leu2 ura3 his 4 can1) and diploid IH1788 strains (MAT a/a trp1 leu2 ura 3 his 4 can1) (Michaelis and Herskowitz, 1988 ) were also used. YPD medium was used for propagation and SD medium, with appropriate nutrients, for selection of transformants. Recombinant plasmids were cloned and propagated in Escherichia coli MC1061 strain (Clontech Laboratories, Inc.) using standard conditions (Sambrook et al., 1989).

\section{Construction of recombinant plasmids}

Primers for PCR amplification of mat A-2 and mat $A-3$ genes were designed using the mat $A$ idiomorph sequence (GenBank M33876); restriction enzyme sites were included at their 5 ' ends to facilitate cloning (Table 1). Amplification reactions performed in a PT-100 thermal cycler
(MJ Research, Inc.) were set up in a final volume of $20 \mu \mathrm{L}$ using primers (Imprint do Brazil Ltda, Campinas, Brazil) and one unit of $\mathrm{Taq}$ polymerase (Phoneutria Biotecnologia \& Serviços, Belo Horizonte, Brazil) in a standard protocol. Plasmid pRAUW84 (Aramayo and Metzenberg, 1996), which has the Nsi I/Nde I mat A idiomorph fragment containing all three mat $A$ genes, was used as template.

Plasmids pGBT9, pGAD424, pVA3, pCL1 and pTD1 were obtained from Clontech Laboratories, Inc.. The pGBT9, pGAD424 plasmids were used for cloning the PCR fragments for the Y2H experiments. pGBT9 contains the GAL4 DNA-binding domain (BD) sequence and the TRP1 gene as selectable marker, while pGAD424 contains the GAL4 activation domain (AD) sequence and the LEU2 gene as selectable marker. For the cloning of the mat $A-3$ amplicon, both plasmids were restriction digested and treated with calf intestinal alkaline phosphatase (Promega Corp., Madison, WI, USA) after digestion for dephosphorylation of 5 ' overhangs. $N$. crassa mat $A-2$ and mat $A-3$ amplicons were purified and digested with the appropriate enzymes for in phase cloning into pGBT9 and pGAD424. Correct cloning was confirmed by PCR using BONO and EDGE primer pairs and restriction analysis.

\section{Two-hybrid experiments}

Yeast cells were transformed following the protocol of Schiestl and Gietz (1989). Transformants were selected by screening for tryptophan, leucine or tryptophan/leucine prototrophy and transferred to SD with necessary requirements. To reduce the number of false positives in the yeast assays, the LacZ and the HIS3 reporter genes were tested. The X-gal filter assay, to detect $L a c Z$ expression, was performed based on the protocol described in the MATCHMAKER Random Peptide Library User Manual PT3039-1 (Clontech Laboratories, Inc.) with modifications. Y190 transformants were grown on sterilized filters on solid SD media without tryptophan, leucine or neither amino acids for three days. Filters with yeast colonies were transferred to clean, sterilized plates and yeast cells were lysed using liquid nitrogen followed by thawing at room temperature. When filters were wet, a solution containing $2.81 \mathrm{~mL}$ of Z-buffer $\left(60 \mathrm{mM} \mathrm{Na}_{2} \mathrm{HPO}_{4}, 40 \mathrm{mM} \mathrm{NaH}_{2} \mathrm{PO}_{4}\right.$, $\left.10 \mathrm{mM} \mathrm{KCl}, 1 \mathrm{mM} \mathrm{MgSO}_{4}\right), 7.6 \mu \mathrm{L}$ of $\beta$-mercaptoethanol and $26.4 \mu \mathrm{L}$ of X-gal $(20 \mathrm{mg} / \mathrm{mL})$ per plate was poured over the filters and the plates were incubated at $37^{\circ} \mathrm{C}$ for $4-16 \mathrm{~h}$.

Table 1 - Sequences of mat $A-2$ and mat $A-3$ primers used in this study.

\begin{tabular}{lll}
\hline Primer & Sequence $\left(5^{\prime}-3^{\prime}\right)$ & Gene \\
\hline EDGE-F & CAGAATTCGACATCAATCTTCTCAACATGCAA & mat $A-2$ \\
EDGE-R & TTGCTGCAGCCACGGATTCTACCATCCCA & mat $A-2$ \\
BONO-F & GTGAATTCCGTCTGCACCCCTCTATCACA & mat $A-3$ \\
BONO-R2 & GTGAATTCTTGGTTGTTGATCAACT & mat $A-3$ \\
\hline
\end{tabular}

Restriction enzyme sites are underlined. GAATTC - EcoR I, CTGCAG - Pst I. 
Cleavage of X-gal leaves a blue color on and around the yeast colonies, being a good indicator of $L a c Z$ gene expression. For the HIS3 reporter assay, HF7c transformants were grown on solid SD media without tryptophan, leucine, or both amino acids, and without histidine. An inhibitor of the enzyme encoded by the HIS3 gene, 3-amino-1,2,4-triazole (3-AT), was used at $2.5 \mathrm{mM}, 5 \mathrm{mM}, 10 \mathrm{mM}$ and $25 \mathrm{mM}$. This technique (which from now on we will call "3-AT assay") allows observing whether there was activation of the HIS3 reporter gene and helps to determine the strength of the activation. Cultures were diluted at $10^{-2}, 10^{-3}$ and $10^{-4}$ and spotted on plates. Plates were incubated at $30{ }^{\circ} \mathrm{C}$ for $5-7$ days.

Y190 and HF7c cells transformed with plasmids pGBT9 and pGAD424, respectively, were used as negative control of activation. Plasmid pVA3, containing the $p 53$ gene lacking its transcriptional activation domain fused to the GAL4p-BD, was used as the control for no transcription activation. Other control transformants bore: plasmid pCL1 which contains the whole $S$. cerevisiae GAL4 gene and is thus a strong activator; and pNTMKad4 (Rodrigues LB, MSc Thesis, Universidade Federal de Minas Gerais, Belo Horizonte, MG, Brazil, 2001), containing a mutated form of the mTEAD1 transcriptional activator, a weak activator. pTD1 has the SV40 large T antigen gene fused to GAL4p$\mathrm{AD}$ and was used in combination with $\mathrm{pVA} 3$ as the positive control for protein-protein interaction in the X-gal assay. Plasmid pCL1 was used as positive control for the HF7c strain in the mono-hybrid experiments.

\section{Growth curves}

The different Y190 transformants were grown overnight at $30{ }^{\circ} \mathrm{C}$ in an orbital shaker in $5 \mathrm{~mL}$ of selective liquid SD media. They were then re-inoculated in $5 \mathrm{~mL}$ of the same medium at $1 \times 10^{6}$ cells $/ \mathrm{mL}$ and grown for two hours in a shaker $(150 \mathrm{rpm})$ at $30^{\circ} \mathrm{C}$. At first, the same growth conditions were used for HF7c transformants; but, due to the extended lag phase of the latter (see results), the re-inoculation step was modified as follows: HF7c transformants were grown overnight at $30{ }^{\circ} \mathrm{C}$ in an orbital shaker (150 rpm) and cells were counted. HF7c/pGBT9 and HF7c/pBDA-3 transformants were inoculated at $0.5 \times 10^{4}$ cells/mL. HF7c/pBDA-2 and HF7c/pBDA-2/ pADA-3 transformants were inoculated at $0.5 \times 10^{5}$ cells $/ \mathrm{mL}$. All inocula were set up in $5 \mathrm{~mL}$ of selective liquid media lacking selected amino acids. Cultures were grown at $30^{\circ} \mathrm{C}$ under $150 \mathrm{rpm}$ for $16 \mathrm{~h}$. Cells were counted at intervals of two hours in a hemocytometer until obtaining four points in the exponential growth curve. Each growth curve was repeated at least three times. The first point of the exponential growth curve was considered as time zero. The mean doubling time of the transformants was calculated using the inclination of the hypothetical exponential growth curves. Graphs were drawn using the Origin 7.5 software (OriginLab Corporation). Statistical analyses were performed using Student's $t$-test at $\mathrm{p}<0.05$ using the Statistica program.

\section{Plasmid cure experiments}

pGBT9, pBDA2, pBDA3 and pBDA2/pADA3 transformants were grown on YPD for $48 \mathrm{~h}$. Then, cells were counted and diluted to attain $300 \mathrm{CFU}$ in an aliquot of $100 \mu \mathrm{L}$ of each culture that were spread over YPD and selective SD plates. SD medium lacked histidine, leucine and adenine. Plates were incubated at $30{ }^{\circ} \mathrm{C}$ and colonies were counted after $72 \mathrm{~h}$. Relative survival was calculated and shown as percentage.

\section{Results and Discussion}

Potential transcriptional activation or silencing of a gene can be detected in yeast reporter gene systems when they are fused to the GAL4 DNA-binding domain (Fields and Song, 1989). N. crassa mat $A-2$ and mat $A-3$ gene products are thought to be transcription factors (Ferreira et al., 1996) and since their description, biochemical data such as those from DNA binding assays have not been reported. Thus to investigate the biochemical functions of the mat A-2 and mat A-3 proteins we decided to use the yeast reporter system. Both mat $A-2$ and mat $A-3$ sequences were cloned into plasmid pGBT9 fused to the $S$. cerevisiae GAL4p DNA-binding domain.

Transformants carrying pBDA-2 (GAL4-BD-mat A2) failed to show reporter gene expression in the two systems, as shown by the lack of growth in medium without histidine and no production of blue colonies (Figures 1B and 2D, respectively). This indicates that mat $A-2$ does not act as an activator of the systems by itself. On the other hand, pBDA-3 (GAL4-BD-mat $A$-3) transformants exhibited some transcriptional activation of both reporter genes (Figures 1B, 1C and 2E). The results suggest a weak activation function for mat $A-3$ in yeast. The mat $A-3$ gene fused with $G A L 4-B D$ led to the growth of HF7c transformants in SD media devoid of tryptophan and histidine (Figure 1B). However, these transformants were unable to grow on the same medium enriched with $2.5 \mathrm{mM} \mathrm{3-AT} \mathrm{(Figure} \mathrm{1C).}$ The assay with the Y190 transformants carrying the mat $A-3$ sequence fused to GAL4p-BD showed a weak $\beta$-galactosidase activity in the filter assay (Figure 2E), which supports the proposed transcriptional activity function for mat A-3.

Indication of transcriptional activation in the yeast reporter system was previously shown for $N$. crassa mat a-1, mat A-1 and mat A-3 proteins (Badgett and Staben, 1999). Using the yeast two-hybrid technique, Jacobsen et al. (2002) showed transcriptional activity of $S$. macrospora SMTA-1 and SMTa-1 mat proteins. However, the homologues of $N$. crassa mat $A-2$ and mat $A-3$ present in $S$. macrospora (SmtA-2 and $S m t A-3$, respectively) did not show any yeast reporter gene activation. The $S m t A-3$ gene 


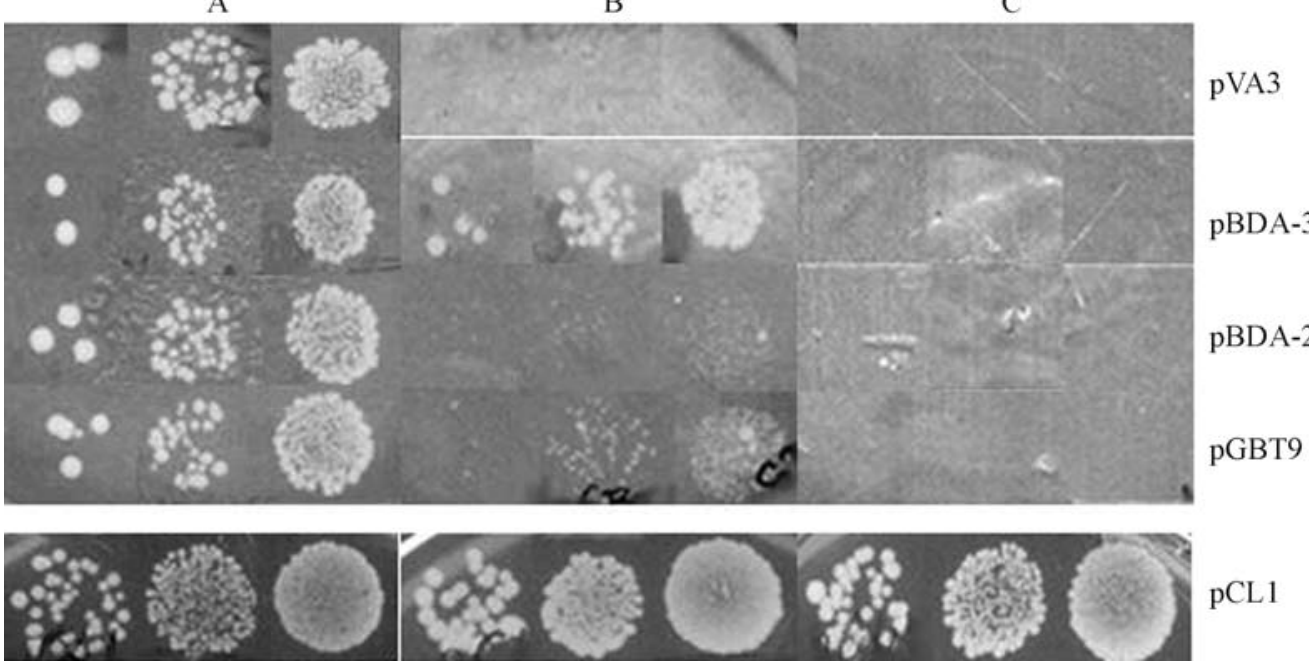

Figure 1 - Transcriptional activation in 3-AT assay. HF7c transformant cultures carrying plasmids pVA3 (pGBT9-p53), pBDA-3 (GAL4-BD-mat A-3), pBDA-2 (GAL4-BD-mat A-2), pGBT9 (vector) or pCL1 (entire GAL4), were spotted, at $10^{-4}, 10^{-3}$ and $10^{-2}$ dilutions of a stationary culture, on SD medium lacking: A) tryptophan (for pVA3, pBDA-3, pBDA-2 and pGBT9) or leucine (for pCL1), B) tryptophan and histidine or leucine and histidine and C) tryptophan and histidine or leucine and histidine and both enriched with $2.5 \mathrm{mM} 3-\mathrm{AT}$.

has high similarity to the $5^{\prime}$ end of the $N$. crassa mat $A-3$ gene and its 3 ' end is more similar to the $N$. crassa mat a idiomorph and does not include the HMG domain (Pöggeler et al., 1997). It is probably due to these differences that the two related proteins show a different behavior in the yeast reporter gene system. The homologue of mat $A-3$ in P. anserina (the SMR2 gene) encodes a protein which, along with FMR1, seems to function as a repressor of the mat+ and as an activator of mat- specific functions (Arnaise et al., 2001). Based on the sequence similarity of $N$. crassa mat $A-3$ with $P$. anserina $S M R 2$ this hypothesis supports the potential gene activation observed for the mat A-3 protein (Ferreira et al., 1996).

Since mat A-2 did not show any transcriptional activity and mat A-3 showed only a weak activation separately, it is possible that an interaction of the mat proteins is necessary for activation or repression of transcription, as suggested by in vivo analysis (Ferreira et al., 1998). In $S$. cerevisiae, $\mathbf{a} 1$ and $\alpha 2$ proteins heterodimerize in the diploid cells, turning off the expression of $\alpha 1$, which is a positive regulator of $\alpha$-specific genes, and of several haploid-specific genes (reviewed in Souza et al., 2003). Heterodimerization of mating-type proteins is also observed in Basidiomycete fungi and the regulatory heterodimer formed is responsible for intracellular recognition of sexual compatibility (reviewed in Bakkeren et al., 2008). Initially, Glass and Staben (1990) postulated a model for N. crassa mat genes function where, in the vegetative phase, they regulate A- and a-specific genes along with those involved with vegetative incompatibility. When fertilization occurs, the mat proteins would interact to form a regulatory activator of the perithecium-differentiation genes. Genetic and RNA analysis of mat mutants also indicate that mat proteins form a complex (Ferreira et al. 1998).
Plasmids pADA-2 (GAL4-AD-mat $A$-2) and pADA-3 (GAL4-AD-mat A-3) were constructed for the two-hybrid analysis. Both plasmids were transformed into yeast in different combinations with plasmids pBDA-2 (GAL4-BDmat $A-2$ ) and pBDA-3 (GAL4-BD-mat A-3). This would allow identifying hetero or homodimerization of $N$. crassa mat A-2 and mat A-3 proteins. Since the $N$. crassa mat proteins were not supposed to bind to the GAL1 promoter sequence, which is the promoter used for the reporter genes, HF7c and Y190 strains were transformed with pADA-2 or pADA-3 to serve as negative control. HF7c and Y190 transformants were then analyzed for expression of their respective reporter gene (Figures 3 and 4 ). In the $\mathrm{X}$-gal assay, pBDA-3/pADA-2 (Figure 3F) showed a weak transcriptional activation of the $L a c Z$ reporter gene, indicating weak interaction between mat A-2 and mat A-3 proteins; $p B D A-$ 3/pADA-3 (Figure 3G) resulted in an ambiguous signal, suggesting that a weak mat A-3/mat A-3 interaction may have occurred. This transcriptional activity seemed to be stronger than that observed for the mat $A-3$ sequence alone (see Figure 2E for comparison).

HF7c transformants carrying the combinations pBDA-3/pADA-3 and pBDA-3/ pADA-2 were able to grow on SD media lacking tryptophan and histidine and enriched with $2.5 \mathrm{mM} 3-\mathrm{AT}$; some growth in the presence of $5 \mathrm{mM}$ 3-AT was also observed (Figures 4B-2, 3 and 4). In addition to the suggestion of homodimerization of mat A-3 protein and heterodimerization of mat A-2 and mat A-3 proteins, the 3-AT two-hybrid test also indicated that both interactions are stronger than the transcriptional activation function of the mat A-3 protein alone (see Figure 1C for comparison).

Surprisingly, the pADA-3 transformant (mat A-3 gene fused to GAL4-AD) showed a weak transcriptional ac- 
tivation in the filter assay (Figure 3C) although no growth of HF7c transformed with the same plasmid was observed in SD media lacking histidine (Figure 4A-2). This indicated that the $N$. crassa mat A-3 protein was also capable of binding to DNA, as expected for HMG proteins, but the interaction was not very strong. The HMG domain is a DNAbinding motif present in transcriptional regulator proteins involved in cell differentiation and non-histone elements of the chromatin, including the SRY-family; some members of the HMG family of proteins bind to DNA with low sequence specificity (Grosschedl et al., 1994). Philley and Staben (1994) demonstrated that the HMG sequence of mat

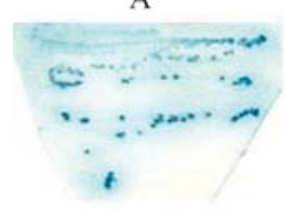

D

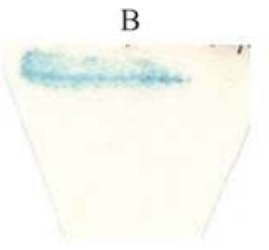

E

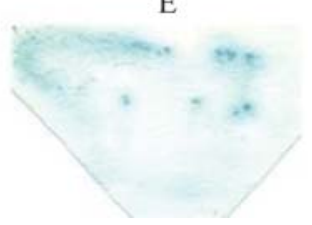

Figure 2 - Transcriptional activation in the X-gal filter assay. Y190 transformant cultures carrying plasmids pCL1 (A; strong transcriptional activator), pNTmKad4 (B; weak transcriptional activator), pGBT9 (C; vector), pBDA-2 (D; GAL4-BD-mat A-2) or pBDA-3 (E; GAL4-BD-mat A-3) were grown on filters laid on solid SD medium lacking tryptophan. Filters were incubated at $37^{\circ} \mathrm{C}$ for $4-16 \mathrm{~h}$. a-1 protein was capable of binding in vitro to the 5'-CTTTG-3' sequence. It was also reported that the mat A-3 protein could bind in vitro to the same sequence (Philley and Staben, unpublished data cited in Ferreira et

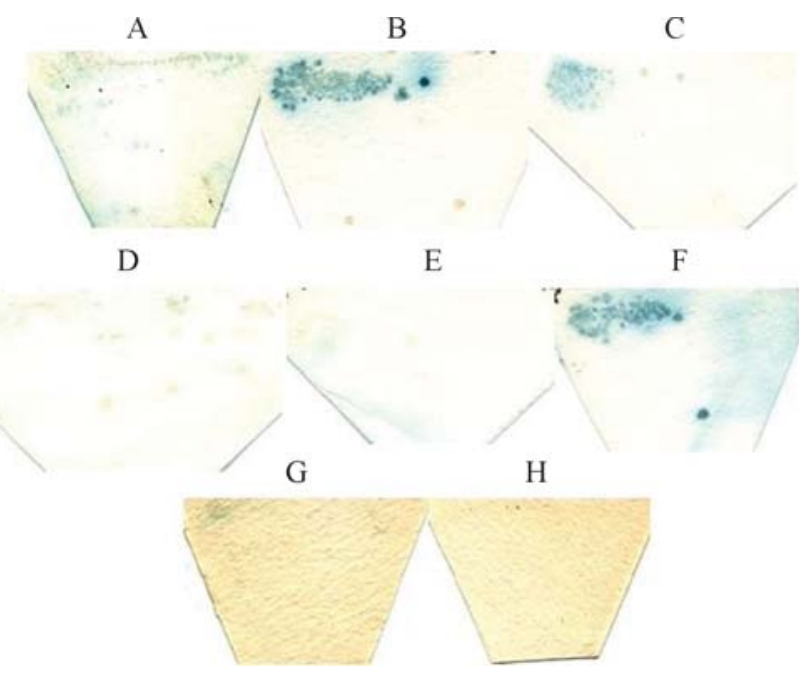

Figure 3 - Two-hybrid X-gal filter assay. Transformant Y190/pGAD424 (A; original plasmid) was used as negative control while transformant Y190/pVA3/pTD1 (B; GAL4-BD-p53/GAL4-AD-sv40 large T antigen) was used as positive control for protein-protein interaction. Y190/pADA-3 (C; GAL4-AD-matA-3) and Y190/pADA-2 (D; GAL4$A D$-mat $A$-2) were used as negative controls for transcriptional activation. These transformants were grown on SD media lacking leucine while Y190/pVA3/pTD1, Y190/pBDA-2 (GAL4-BD-matA-2)/pADA-2 (E), Y190/pBDA-3 (GAL4-BD-mat A-3)/pADA-2 (F), Y190/pBDA3/pADA-3 (G) and Y190/ pBDA-2/pADA-3 (H) were grown on SD media lacking both tryptophan and leucine.

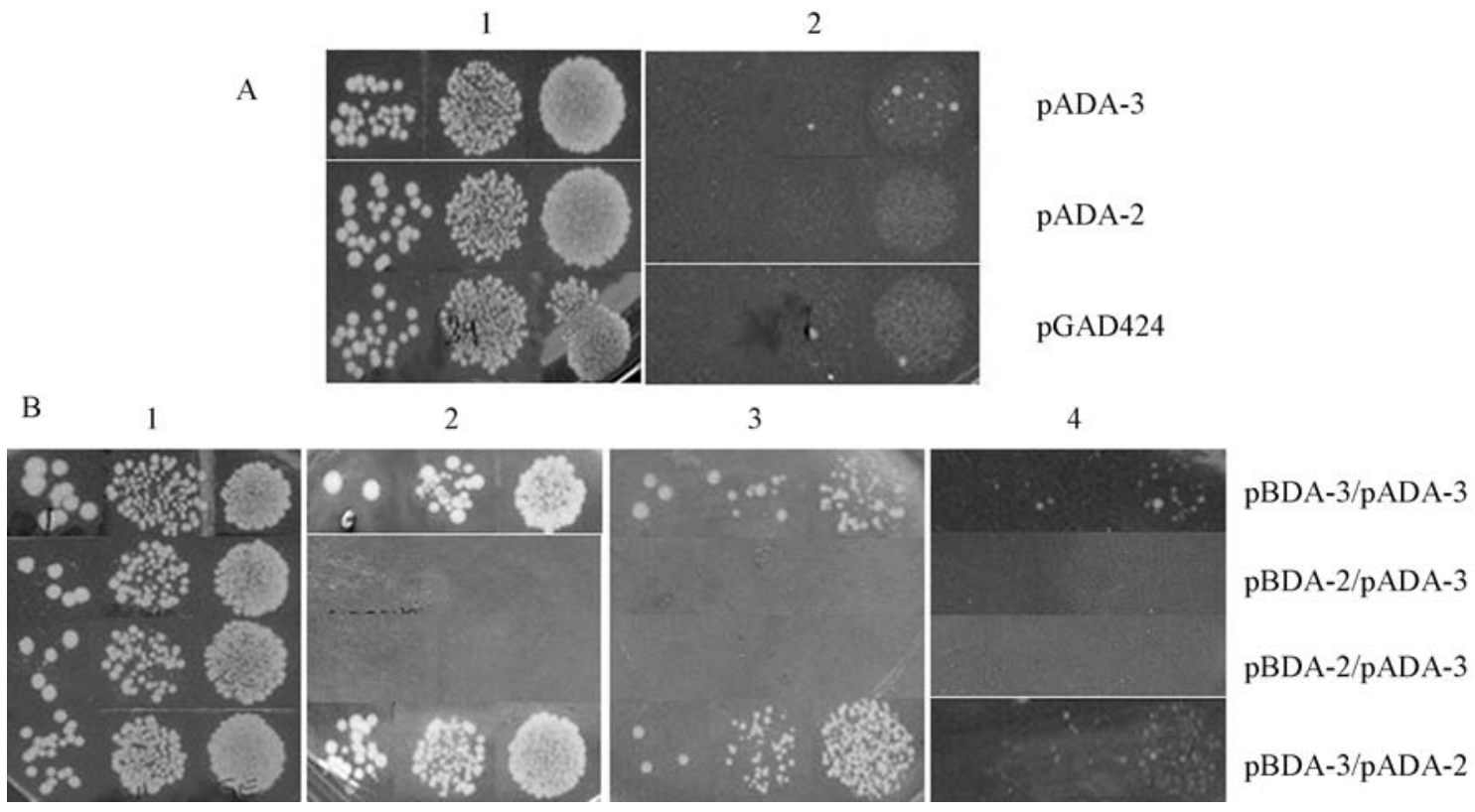

Figure 4 - Two-hybrid 3-AT assay. Drops of HF7c transformant cultures carrying plasmids pADA-3 (GAL4-AD-mat A-3), pADA-2 (GAL4-AD-mat $A-2$ ), pGAD424 (vector) (A), and combinations pBDA-3 (GAL4-BD-mat A-3)/pADA-3, pBDA-2 (GAL4-BD-mat A-2)/pADA-3, pBDA-2/pADA-2 and pBDA-3/pADA-2 (B) were applied on SD solid media. A) SD media devoid of leucine (1), for control purpose, and devoid of leucine and histidine (2). B) SD media devoid of leucine and tryptophan (control) (1); leucine, tryptophan and histidine (2); leucine, tryptophan and histidine enriched with $2.5 \mathrm{mM}$ 3-AT (3) and 5 mM 3-AT (4). 
al., 1996). The 907 bp sequence of the GAL1 promoter (GenBank K02115) contains a unique 5'-CTTTG-3' sequence at position 121 and its complement (5'-CAAAG-3') at position 186. These are not included in the $G A L 1_{U A S}$ sequence (298-663 bp region) (Guarente et al., 1982; Yocum et al., 1984; GenBank K02115) which is the promoter used for the HIS3 reporter in the HF7c and the lacZ reporter in Y190. However, there are at least three 5'-A/TAACAAT/A-3' sequences, that are known to be DNA binding motifs for HMG proteins in the GAL1 $1_{U A S}$ sequence. It is possible that the HMG domain of the mat A-3 protein recognized one or more of these DNA-binding sites in the $G A L 1_{U A S}$ promoter with low sequence specificity.

Not even a low transcription level of the HIS3 reporter gene was observed in HF7c cells transformed with pADA-3 (GAL4p-mat $A-3)$. Since we are assuming that the mat A-3 protein interacted with low specificity with the DNA-binding sites present in the GAL1 promoter, the absence of growth in the HF7c transformant probably occurred because the interaction was not strong enough to activate transcription to a level that could allow growth of the transformant in SD - his.

In our two-hybrid experiments, a weak interaction between mat A-2 and mat A-3 was observed when these proteins were fused to GAL4p-AD and GAL4p-BD domains, respectively. It may be that the proper folding of mat A-2 and/or mat A-3 was not established when the fusion was made with GAL4p-BD and GAL4p-AD, respectively. Our result, along with that of Badgett and Staben (1999), supports the hypothesis of mating-type proteins working in a complex to activate or repress genes in different developmental pathways (Ferreira et al., 1998). Curiously, in $P$. anserina protein-protein interaction in two-hybrid assays was only seen between the FMR1 and SMR2 proteins (Debuchy and Coppin, unpublished data cited in Coppin et al., 1997). It is known that all four $P$. anserina mat genes are necessary for the post-fertilization events (Debuchy et al., 1993); and FPR1 and the heterodimer FMR1/SMR2 are responsible for internuclear recognition, while SMR1 protein is required for dikaryotic cell formation (Zickler et al., 1995; Arnaise et al., 1997). The mat A-2 and mat A-3 proteins are most likely both involved with multiple nuclei mitotic divisions before migration into the crozier, since the few progeny observed in crosses with the double mutants is totally biparental (Ferreira et al., 1998). Thus, our results support the idea that $N$. crassa and $P$. anserina differ from one another in the control of post-fertilization events, as was postulated in previous studies (Ferreira et al., 1996, 1998).

Expression of some proteins in yeast can cause a toxic phenotype which may be detected by growth retardation and even death (Nonaka et al., 2000; Roopchand et al., 2001; Blanco et al., 2003; Boyer et al., 2004; Buryskova et al., 2004). During our experiments, pBDA-2 (GAL4p-BDmat $A-2)$ and pBDA-2/pADA-3 (GAL4p-AD-mat $A-3)$ transformants grew slower than other transformants. In addition, a new transformation with the pBDA-2/pADA-3 into HF7c cells had to be performed three times for completing the experiments, since the transformants survived less than four weeks in solid media. This is a rather short time compared to the other transformants which were viable for over a month under the same conditions. Also, the transformation frequency was always lower with pBDA-2 (not shown). It was thus hypothesized that the pBDA-2 plasmid could be toxic to yeast cells. In an attempt to test this hypothesis, growth of these transformants was analyzed over time.

The lag phase of both pBDA-2 and pBDA-2/pADA-3 transformants was longer, taking at least two additional hours before they achieved the log phase (not shown). This was more noticeable in the HF7c background which normally grows slower than the Y190 strain (data not shown). Conversely, the pBDA-3 transformants showed the shortest lag phase. At this phase, the metabolism of yeast cells changes in order to maximally benefit from the new environment. This is manifest in the strongly enhanced expression of proteins involved in the metabolism of carbohydrate and amino acids as well as protein synthesis (Brejning et al., 2003). It is possible that the presence of mat A-2 protein fused to the GAL4p-BD domain interferes with the adaptation of the yeast cells to the new environment. This could explain the increase in the lag phase observed in transformants carrying plasmid pBDA-2. The mat A-3 protein fused to the GAL4p-BD domain, conversely, could be helping the yeast cells in this adaptation, thus shortening the lag phase.

Although the lag phase was increased in all mat A-2 transformants, no significant difference was observed at the exponential phase, as indicated by the cell doubling time $(p>0.05)$. No difference in doubling time was detected between HF7c transformants bearing pBDA-2 and pBDA2/pADA-3. However, the doubling time of these two transformants is longer than the one observed for the HF7c pGBT9 transformants (Table 2).

Being interested in the interference of $N$. crassa mating proteins on the growth of $S$. cerevisiae, we conducted a plasmid cure experiment. Y190 cells harboring the four different constructs were grown without selective pressure (YPD) and then plated on SD lacking the specific marker amino acids and on YPD plates as control (Table 2). Ninety to $100 \%$ of the plated pGBT9 transformants were able to grow on selective media. Transformants harboring mat A-3 also had a high survival rate (68\%-72\%). However, cells initially harboring the mat A-2 gene, either alone or in combination with mat A-3, showed a dramatic loss of the markers in the absence of selective pressure (4.8\%-13.2\%), indicating a toxic effect of this construct on yeast.

The N. crassa mating-type proteins that were expressed in $S$. cerevisiae in this study are thought to be developmentally regulated and only functional in the sexual 
Table 2 - Plasmid cure in Y190 cells.

\begin{tabular}{lcccc}
\hline & \multicolumn{4}{c}{ Number of colonies $^{\mathrm{a}}$} \\
\cline { 2 - 5 } Media & pGBT9 & pBDA-2 & pBDA-3 & pBDA-2/pADA-3 \\
\hline YPD & $189-208$ & $165-242$ & $161-288$ & $156-280$ \\
SD -aa & $307^{* *}-188$ & $8-32$ & $116-195$ & $2-13$ \\
\hline
\end{tabular}

${ }^{a}$ Number of colonies per plate in two different experiments. **High number of colonies in this control may be due to plating error. pGBT9 - vector, pBDA-2 - GAL4-BD-mat A-2, pBDA-3 - GAL4-BD-mat A-3, pADA-3 - GAL4-AD-mat A-3.

Table 3 - Doubling time of different yeast cells transformed with pGBT9, pBDA-2 and pBDA-3.

\begin{tabular}{lcccc}
\hline Strain & \multicolumn{4}{c}{ Cell doubling time (mean in minutes) } \\
\cline { 2 - 5 } & pGBT9 & pBDA-2 & pBDA-3 & pBDA-2/pADA-3 \\
\hline HF7c & 151 & 183 & 147 & 200 \\
Y190 & 112.5 & 129 & 116 & 185 \\
IH1783(a) & 135 & 124 & 116 & - \\
IH1784 $(\alpha)$ & 153 & 191 & 132 & - \\
IH1788 $(\mathrm{a} / \alpha)$ & 140 & 169 & 123 & - \\
\hline
\end{tabular}

pGBT9 - vector, pBDA-2 - GAL4-BD-mat A-2, pBDA-3 - GAL4-BD-mat A-3, pADA-3 - GAL4-AD-mat A-3.

cycle (Ferreira et al., 1996). Our initial experiments were performed using only the mitotic cell cycle of this yeast, and thus both mat proteins were expressed during a cell cycle in which they normally would be silenced. Although mat A-2 and mat A-3 do not exhibit a domain similar to the MAT proteins of $S$. cerevisiae we wondered whether the mating-type and ploidy state of the yeast receiver would make any difference in the growth of the transformants. Plasmids pGBT9, pBDA-2, pDBA-3 were transformed into isogenic haploid (IH1783a and IH 1784 $\alpha$ ) and diploid cells (IH1788a/ $\alpha$ ). These transformants were grown on selective media and their growth rates were measured (Table 3). The ploidy apparently did not make any difference since the doubling time of the diploid transformant was not affected by the presence of the mat genes $(p>0.05)$. Apparently in the mat $\alpha$ background the doubling times for mat A-2 transformants were higher. However, statistical tests showed no significant difference probably because of the high variance between the repetitions (data not shown). Thus we cannot affirm that $N$. crassa proteins have an effect in the yeast cell cycle.

A curious aspect is that, although mat A-2 and mat A-3 proteins seem to have similar functions in N. crassa (Ferreira et al. 1998), they displayed different behavior in the two-hybrid experiment. Nevertheless, a more clear understanding about the roles of the mat A-2 and mat A-3 proteins can only be reached when their target genes are identified. Galgoczy et al. (2004) were able to identify a large number of specific target genes of $S$. cerevisiae mat proteins using genome-wide chromatin immunoprecipitation, transcriptional profiling and phylogenetic comparisons. Different studies aiming at analyzing mat-regulated genes in sexual and non-sexual fungi demonstrate the importance of understanding the control of vegetative and sexual cycles in filamentous fungi (Nowrousian et al., 2005; Pögeller et al. 2006; Keszthelyi et al., 2007). In this context, the pattern of interaction of mat proteins studied here contributes towards understanding the control of vegetative and sexual cycles in $N$. crassa and in other fungal species.

\section{Acknowledgments}

The authors wish to thank CAPES for the graduate scholarships to CC da Silva and RC Cruz.

\section{References}

Aramayo R and Metzenberg RL (1996) Meiotic transvection in fungi. Cell 86:103-113.

Arnaise S, Zickler D and Glass NL (1993) Heterologous expression of mating-type genes in filamentous fungi. Proc Natl Acad Sci USA 90:616-620.

Arnaise S, Debuchy R and Picard M (1997) What is a bono fide mating-type gene? Internuclear complementation of mat mutants in Podospora anserina. Mol Gen Genet 256:169178.

Arnaise S, Zickler D, Bilcot SL, Poisier C and Debuchy R (2001) Mutations in mating-type of the heterothallic fungus Podospora anserina lead to self-fertility. Genetics 159:545-556.

Badgett TC and Staben C (1999) Interaction between and transactivation by mating type polypeptides of Neurospora crassa. Proceedings of Fungal Genetics Conference 20:127.

Bakkeren G, Kämper J and Schirawski J (2008) Sex in smut fungi: Structure, function and evolution of mating-type complexes. Fungal Genet Biol 45:S15-S21.

Blanco R, Carrasco L and Ventoso I (2003) Cell killing by HIV-1 protease. J Biol Chem 278:1086-1093. 
Bobrowicz P, Pawlak R, Correa A, Bell-Pedersen D and Ebbole DJ (2002) The Neurospora crassa pheromone precursor genes are regulated by the mating type locus and the circadian clock. Mol Microbiol 45:795-804.

Boyer J, Badis G, Fairhead C, Talla E, Hantraye F, Badis G, Fairhead C, Talla E, Hantraye F, Fabre E et al. (2004) Large-scale exploration of growth inhibition caused by overexpression of genomic fragments in Saccharomyces cerevisiae. Genome Biol 5:R75.1-R75.19.

Brejning J, Jespersen L and Arneborg N (2003) Genome-wide transcriptional changes during the lag phase of Saccharomyces cerevisiae. Arch Microbiol 179:278-294.

Buryskova M, Pospisek M, Grothey A, Simmet T and Burysek L (2004) Intercellular interleukin- $1 \alpha$ functionally interacts with histone acetyltransferase complexes. J Biol Chem 279:4017-4026.

Coppin E, Debuchy R, Arnaise S and Picard M (1997) Mating types and sexual development in filamentous ascomycetes. Microbiol Mol Biol Rev 61:411-428.

Debuchy R, Arnaise S and Lecellier G (1993) The mat- allele of Podospora anserina contains three regulatory genes required for the development of fertilized female organs. Mol Gen Genet 241:667-673.

Ferreira AV-B, Saupe S and Glass NL (1996) Transcriptional analysis of the $m t A$ idiomorph of Neurospora crassa identifies two genes in addition to $m t A-1$. Mol Gen Genet 250:767-774.

Ferreira AV-B, An Z, Metzenberg RL and Glass NL (1998) Characterization of mat $A-2$, mat $A-3$ and $\triangle$ matA mating-type mutants of Neurospora crassa. Genetics 148:1069-1079.

Fields S and Song O (1989) A novel genetic system to detect protein-protein interactions. Nature 340:245-246.

Galgoczy DJ, Cassidy-Stone A, Llinás M, O'Rourke S, Herskowitz I, DeRisi JL and Johnson AD (2004) Genomic dissection of the cell-type-specification circuit in Saccharomyces cerevisiae. Proc Natl Acad Sci USA 101:18069-18074.

Glass NL and Staben C (1990) Genetic control of mating in Neurospora crassa. Dev Biol 1:177-184.

Glass NL, Vollmer SJ, Staben C, Grotelueschen J, Metzenberg RL and Yanofsky C (1988) DNAs of the two mating-type alleles of Neurospora crassa are highly dissimilar. Science 241:570-573.

Glass NL, Grotelueschen J and Metzenberg RL (1990) Neurospora crassa A mating-type region. Proc Natl Acad Sci USA 87:4912-4916.

Grosschedl R, Giese K and Pagel J (1994) HMG domain proteins: Architectural elements in the assembly of nucleoprotein structures. Trends Genet 10:94-100.

Guarente L, Yocum RR and Giffor P (1982) A GAL10-CYC1 hybrid yeast promoter identifies the GAL4 regulatory region as an upstream site. Proc Natl Acad Sci USA 79:7410-7414.

Jacobsen S, Wittig M and Pöggeler S (2002) Interaction between mating-type proteins from the homothallic fungus Sordaria macrospora. Curr Genet 41:150-158.

Keszthelyi A, Jeney A, Kerényi Z, Mendes O, Waalwijk C and Hornok L (2007) Tagging target genes of the MAT1-2-1 transcription factor in Fusarium verticilloides (Gibberella fujikuroi MP-A). Antonie Van Leeuwenhoek 91:373-391.
Kim H, Metzenberg RL and Nelson MA (2002) Multiple functions of mfa-1, a putative pheromone precursor gene of Neurospora crassa. Eukaryot Cell 1:987-999.

Kronstad JW and Staben C (1997) Mating type in filamentous fungi. Annu Rev Genet 31:245-276.

Metzenberg RL and Glass NL (1990) Mating type and mating strategies in Neurospora. BioEssays 12:53-59.

Michaelis S and Herskowitz I (1988) The a-factor pheromone of Saccharomyces cerevisiae is essential for mating. Mol Cell Biol 8:1309-1318.

Nonaka G, Ishikawa T, Liu T and Nakajima H (2000) Genetic analysis of growth inhibition of yeast cells caused by expression of Aspergillus oryzae RNase T1. Biosci Biotechnol Biochem 64:2152-2158.

Nowrousian M, Ringelberg C, Dunlap JC, Loros JJ and Kück U (2005) Cross-species microarray hybridization to identify developmentally regulated genes in the filamentous fungus Sordaria macrospora. Mol Genet Genomics 273:137-149.

Philley ML and Staben C (1994) Functional analyses of the Neurospora crassa MT a-1 mating-type polypeptide. Genetics 137:715-722.

Pöggeler S and Kück U (2000) Comparative analysis of the mating-type loci from Neurospora crassa and Sordaria macrospora: Identification of novel transcribed ORFs. Mol Gen Genet 263:292-301.

Pöggeler S, Risch S, Kück U and Osiewacz HD (1997) Matingtype genes from the homothallic fungus Sordaria macrospora are functionally expressed in a heterothallic ascomycete. Genetics 147:567-580.

Pöggeler S, Nowrousian M, Ringelberg C, Loros JJ, Dunlap JC and Kück U (2006) Microarray and real time PCR analysis reveal mating type-dependent gene expression in a homothallic fungus. Mol Genet Genomics 275:492-503.

Roopchand DE, Lee JM, Shahinian S, Paquette D, Bussey H and Branton PE (2001) Toxicity of human adenovirus E4orf4 protein in Saccharomyces cerevisiae results from interactions with Cdc55 regulatory B subunit of PP2A. Oncogene 20:5279-5290.

Sambrook J, Fritsch EF and Maniatis T (1989) Molecular Cloning: A Laboratory Manual. 2nd edition. Cold Spring Harbor Laboratory Press, Cold Spring Harbor.

Schiestl RH and Gietz RD (1989) High efficiency transformation of intact yeast cells using single stranded nuclei acids as carrier. Curr Genet 16:339-346.

Souza CAJ, Silva CC and Ferreira AV-B (2003) Sex in fungi: Lessons of gene regulation. Genet Mol Res 2:136-147.

Staben C and Yanofsky C (1990) Neurospora crassa a mating-type region. Proc Natl Acad Sci USA 87:4917-4921.

Yocum RR, Hanley S, West Jr. R and Ptashne M (1984) Use of $\mathrm{LacZ}$ fusions to delimit regulatory elements of the inducible divergent GAL1-GAL10 promoter in Saccharomyces cerevisiae. Mol Cell Biol 4:1985-1998.

Zickler D, Arnaise S, Coppin E, Debuchy R and Picard M (1995) Altered mating-type identity in the fungus Podospora anserina leads to selfish nuclei, uniparental progeny and haploid meiosis. Genetics 140:493-503.

Associate Editor: Sérgio Olavo Pinto da Costa

License information: This is an open-access article distributed under the terms of the Creative Commons Attribution License, which permits unrestricted use, distribution, and reproduction in any medium, provided the original work is properly cited. 